\title{
PROJECT MUSE'
}

De la loi Toubon à la loi Fioraso: Quel cadre légal pour

les formations en anglais dans les universités françaises

?

Jean-François Graziani

European Journal of Language Policy, Volume 6, Issue 2, Autumn 2014, pp. 159-174 (Article)

Published by Liverpool University Press

$\Rightarrow$ For additional information about this article https://muse.jhu.edu/article/565542 


\title{
De la loi Toubon à la loi Fioraso
}

\section{Quel cadre légal pour les formations en anglais dans les universités françaises?}

\author{
Jean-François Graziani \\ Université d'Osaka \\ jeffgraz@hotmail.com
}

\begin{abstract}
Résumé
La loi du 4 août 1994 relative à l'emploi de la langue française, dite loi Toubon, a été votée pour protéger les consommateurs, les travailleurs et la création culturelle en langue française face à la prédominance croissante de l'anglais et de la culture américaine en Europe. En droit, son domaine d'application s'étend aussi à l'enseignement. En avril et mai 2013, Geneviève Fioraso, ministre de l'Enseignement Supérieur et de la Recherche, a présenté au Sénat et à l'Assemblée un projet de réforme des universités dont le deuxième article a fait couler beaucoup d'encre. En effet, cet article proposait d'aménager la loi Toubon, ou plutôt de la vider de sa substance en élargissant le champ des exceptions, pour permettre aux établissements universitaires français de mettre en place des cours enseignés en « langue étrangère ", c'est-à-dire en anglais. Des difficultés à faire appliquer la loi à la régularisation des établissements en infraction, les évolutions du cadre juridico-linguistique en France illustrent, peut-être de manière plus évidente qu'ailleurs, les problématiques nouvelles auxquelles sont confrontées les universités dans un contexte d'hypercompétition à l'échelle planétaire.
\end{abstract}

Mots clés : cours en anglais ; anglicisation ; langue française ; enseignement supérieur ; loi Toubon ; loi Fioraso

\section{Enseigner en langue étrangère dans les universités françaises}

En avril et mai 2013, l'actualité a été marquée par une polémique que l'on pourrait hâtivement qualifier de "franco-française » mais qui, en réalité, concerne l'ensemble des pays de l'OCDE. Geneviève Fioraso, ministre de l'Enseignement Supérieur et de la Recherche, a présenté au Sénat et à l'Assemblée un projet de réforme des universités dont le deuxième article a fait couler beaucoup d'encre. 
En effet, cet article proposait d'aménager la loi Toubon pour permettre aux établissements universitaires français de mettre en place des cours enseignés en langue étrangère, en toute légalité. La loi Toubon, sous son intitulé officiel de loi du 4 août 1994 relative à l'emploi de la langue française, stipule que le français est la langue de l'enseignement, des examens et des soutenances de thèses dans le supérieur, les seules exceptions admises étant les cours de langues étrangères et de professeurs étrangers, associés ou invités, autorisés à enseigner dans leur langue maternelle. Dans le Code de l'Éducation, l'article L. 121-3, qui découle directement de la loi Toubon, mentionne également les formations à vocation internationale mais ne s'appliquait sur ce point qu'à certaines sections bilingues de collège et lycée. En d'autres termes, l'article 2 du projet de loi sur l'Enseignement Supérieur et la Recherche (ESR) devait permettre d'élargir autant que possible le champ des exceptions à la loi Toubon pour laisser les coudées franches aux universités françaises et leur permettre de mettre en place la politique linguistique de leur choix, en fonction des besoins de leurs étudiants.

L'Académie française a été l'une des premières à réagir, avec une certaine véhémence d'ailleurs, dénonçant une formulation trop vague de la loi qui risquait ainsi de favoriser " une marginalisation de notre langue " (Déclaration du 21 mars 2013). Des écrivains et des intellectuels comme Antoine Compagnon (2013) et Claude Hagège (Arnaud 2013), professeurs au Collège de France, Bernard Cerquiglini (2013), recteur de l'Agence universitaire de la Francophonie ou le philosophe Michel Serres (Arnaud 2013) ont manifesté dans la presse leur opposition à cet assouplissement du cadre juridique, perçu comme une menace directe pour la langue française. Ils s'inquiètent du très mauvais signal envoyé aux francophones et aux apprenants de français du monde entier. Le cas de Jacques Attali, qui figure dans cette liste des opposants aux cours enseignés en langue étrangère, est des plus intéressants. L’ancien conseiller de François Mitterrand, économiste et écrivain, est connu pour son libéralisme éclairé. On ne peut guère le soupçonner de dérive passéiste (ou passéisante). Il est pourtant l'auteur d'une tribune particulièrement virulente, intitulée : " Enseigner en français !» (sur son blog hébergé par L'Express, mise en ligne le 22 avril 2013), qui attaque le projet d'aménagement linguistique de l'article 2 dans un langage peu diplomatique : «On ne peut pas imaginer idée plus stupide, plus contreproductive, plus dangereuse et plus contraire à l'intérêt de la France. » Le reste du billet est à l'avenant et développe de manière méthodique les quatre arguments initialement annoncés.

C'est le même Jacques Attali qui dans son rapport de 1998, dans la section II. 6. Accentuer l'ouverture au monde) pour un modèle européen d'enseignement supérieur préconisait alors la mise en place de cours enseignés en anglais : 
Idéalement, la France devra pouvoir devenir partie intégrante naturelle du parcours universitaire des étudiants les plus brillants de tout pays du monde. Pour que la méconnaissance de la langue française ne soit plus un obstacle à la venue d'étudiants étrangers, une partie des enseignements devra être assurée en anglais et au besoin par des enseignants non francophones. (Attali 2013:23)

Faut-il préciser que la citation se retrouve presque textuellement dans l'exposé des motifs de l'article 2 du projet de loi et qu'elle a été abondamment reprise par Geneviève Fioraso au cours des différent entretiens accordés à la presse ? Pour qui s'étonnerait d'une évolution aussi stupéfiante, et aussi un peu pour consoler la ministre qui a dû tomber de haut en découvrant la tribune de Jacques Attali, on se contentera de souligner que dans la temporalité hypervéloce de la mondialisation 15 ans représentent une éternité.

À l'Assemblée, la droite souverainiste et l'extrême gauche se sont retrouvées pour dénoncer ce sacrifice éhonté d'une composante majeure de l'identité nationale aux intérêts mercantiles de la mondialisation. Au sein même de la majorité, une fronde menée par Pouria Amirshahi, député de la ge circonscription des Français établis hors de France (Maghreb/Afrique de l'Ouest) et secrétaire national du parti socialiste (PS) aux transitions démocratiques et à la Francophonie, soutenu par une quarantaine de députés socialistes, a conduit le gouvernement à donner des garanties sur l'encadrement et les limites de l'usage d'une langue autre que le français comme langue d'enseignement dans les établissements universitaires. La loi a finalement été votée par l'Assemblée, le 28 mai 2013, avec 248 voix contre et seulement 289 voix pour, avant de repasser devant le Sénat puis de revenir à l'Assemblée pour son adoption définitive le 9 juillet, mettant un terme (provisoire ?) à un débat souvent passionné et pour le moins inattendu pour une question d'aménagement linguistique. Il suffit pourtant de rappeler que lors d'un autre débat, celui portant sur l'identité nationale en 2009, un sondage avait révélé que 80 pour cent des personnes interrogées considéraient la langue française comme l'élément le plus important pour l'identité de la France, loin devant le drapeau tricolore, la laïcité ou les services publics; et l'on n’a jamais autant cité que ces dernières années, dans tous les médias, ce mot célèbre attribué à Albert Camus : «ma patrie, c'est la langue française ». Le climat autour des questions symboliques et identitaires, en France comme dans beaucoup d'autres pays développés, n'est pas des plus sereins mais comment pourrait-il en être autrement dans un contexte de compétition planétaire, parfois féroce, où même les systèmes d'enseignement ne sont plus épargnés?

Il faut enfin relever un dernier fait important pour la compréhension du contexte dans lequel s'est déroulée cette polémique. En dépit de la formulation 
de l'article 2 du projet de loi ESR, qui parle de «langue étrangère », les discussions ont porté essentiellement sur les avantages et les dangers de formations universitaires dispensées en langue anglaise. Geneviève Fioraso elle-même ne s'en est pas cachée ni dans les interviews accordées dans les médias, ni dans les débats au Sénat ou à l'Assemblée : c'est bien de formations en anglais qu'il s'agit. Personne n'est dupe. ${ }^{1}$ La question posée est évidemment celle-ci : peut-on autoriser des enseignements entièrement dispensés en anglais, autres que des cours de langue, dans les universités françaises ? Et donc aussi, bien sûr, des examens et des soutenances de thèses en anglais? Avec quelles conséquences? En somme, seul le texte de la loi conserve la formule de "langue étrangère ", unaniment traduite par langue anglaise dans le débat public. Certains y voient une forme d'hypocrisie, d'autres qui interprètent cette disposition comme une ouverture des universités à une supposée demande linguistique des étudiants étrangers font remarquer que la situation pourrait, en effet, évoluer en fonction du poids mondial de telle ou telle langue. Aujourd'hui l'anglais, demain le chinois? ${ }^{2}$

\section{Dura lex sed lex : la loi Toubon à l'épreuve de l'internationalisation}

\subsection{La loi Toubon dans l'enseignement supérieur et la recherche}

En 1992, la révision du deuxième article introduit dans la Constitution la déclaration suivante : "La langue de la République est le français. " La loi relative à l'emploi de la langue française du 4 août 1994, dite loi Toubon, du nom du ministre de la Culture et de la Francophonie de l'époque, découle de ce principe constitutionnel dont elle précise le champ et les modalités d'application. Selon Jacques Toubon lui-même, cette loi est avant tout « une loi état d'esprit » (cité par Eloy $1997:$ 15), autrement dit ce n'est pas l'efficacité pratique immédiate qui est recherchée, même si certaines mesures comme l'obligation dans les contrats passés par les personnes morales de droit public d'utiliser les équivalents français de termes anglais créés ou avalisés par les commissions de terminologie et de néologie peuvent laisser penser le contraire, mais elle exprime plutôt une volonté d'affirmer (ou de réaffirmer) solennellement

1. Le 20 mai 2013, à la veille de l'ouverture des débats sur le projet de loi ESR à

l'Assemblée, Libération a fait sa Une tout en anglais, avec en gros titre : « Teaching in English ? Let's do it. »

2. Comme le note malicieusement François Garçon, maître de conférences à l'Université Paris I, dans un article publié sur Le Plus, site du Nouvel Observateur : «Fioraso veut des cours en anglais à la fac : évidemment, c'est une bonne idée » (Garçon 2013 : 16 mai). 
l'attachement de la France à sa langue, à ses valeurs et à ses principes. Il ne semble pas non plus complètement illégitime de vouloir garantir le droit du citoyen français à être informé et à pouvoir travailler dans sa langue maternelle sur le territoire national. L'inflation spectaculaire de termes anglais dans le vocabulaire de professions comme la publicité ou la gestion (le management [mænidzmənt], en fait), qui jouent un rôle d'avant-garde, la diffusion massive de biens culturels américains (chansons, cinéma, séries TV) et la volonté de certaines entreprises d'imposer l'anglais comme langue de travail à tous leurs employés, parfois en l'absence de besoins clairement identifiables, voire au détriment de leur productivité et de leurs propres intérêts (Hagège 2012: 78 ; Truchot $2008: 110-17)$, constituent autant de signes tangibles d'une pression linguistique qui s'exerce sur l'ensemble du corps social.

On peut citer cette anecdote qui en dit long : Jean-Marc Ayrault, premier ministre, irrité par l'usage intempestif de l'anglais au sommet de l'Etat a cru nécessaire d'adresser à ses ministres une circulaire " relative à l'emploi de la langue française » (le 25 avril 2013), évoquant Villers-Cotterêts et la révision constitutionnelle de 1992, soulignant que "notre pays s'est construit dans un rapport étroit à la langue française » et ajoutant que " notre langue est à même d'exprimer toutes les réalités contemporaines et de désigner les innovations qui ne cessent de voir le jour dans les sciences et les techniques ».

En ce qui concerne l'enseignement supérieur et la recherche, l'article premier de la loi Toubon stipule que " [la langue française] est la langue de l'enseignement, du travail, des échanges et des services publics» (Gouvernement français 2014b). On se gardera bien d'en tirer des conclusions hâtives mais on ne peut s'empêcher de remarquer que le législateur a placé l'enseignement au début de cette liste. À cet égard, le Code de l'Éducation, qui regroupe l'ensemble des dispositions législatives et réglementaires relatives au système éducatif national, rappelle, dans l'article L. 121-3, que le français est la langue de l'enseignement, des examens et des soutenances de thèses dans le supérieur (Gouvernement français 2014a). Il précise aussi le champ des exceptions admises parmi lesquelles on trouve les cours de langues étrangères et ceux des professeurs étrangers, associés ou invités, autorisés à enseigner dans leur langue maternelle.

Puisqu'il est question de recherche, il faut également mentionner l'article 6 de la loi Toubon qui concerne les congrès et les colloques organisés en France par une personne physique ou morale de nationalité française, autrement dit la majeure partie des colloques qui ont lieu dans les universités françaises et qui constituent une part importante de l'activité des chercheurs. Larticle 6 garantit le droit de chaque participant de s'exprimer en français, entre 
autres dispositions. Il impose aussi aux personnes morales de droit public ou chargée d'une mission de service public, lorsqu'elles sont à l'origine de ce type de manifestation, de prévoir un dispositif de traduction en français. Le non respect des dispositions de l'article 6 est sanctionné par une contravention de 4ème classe, sur une échelle qui va de la moins grave (ière classe) à la plus grave (5ème classe), une amende de $€ 750$ maximum et peut entraîner le retrait des subventions publiques qui leur ont été allouées. À titre de comparaison, c'est l'équivalent d'un demi-tour sur l'autoroute. En revanche, aucune sanction n'est prévue dans le texte de l'article L 121-3 du Code de l'Éducation. Dans tous les cas, un certain nombre d'associations agréées par l'État (le Droit de comprendre, Défense de la langue française, Avenir de la langue française...) sont autorisées à se porter partie civile en cas de litige.

\subsection{Dérives et infractions: de la non-application à la régularisation}

Sans être excessivement procédurier, on peut tout de même s'étonner de la légèreté avec laquelle les autorités officielles ont traité la question de l'application de la loi Toubon dans les établissements universitaires ces dernières années. Si cette loi a donné lieu à des décisions de justice contre certaines entreprises récalcitrantes, dans l'enseignement supérieur, en revanche, c'est le calme plat. Ainsi le Nouvel Observateur, en plein débat sur la loi ESR, a publié un article intitulé : "Cours $100 \%$ en anglais à la fac : ça se fait déjà, my dear!" (Gonzague 2013). Certains cas de violation avaient déjà été relevés dans un document mis en ligne par Hervé Beaudin (2010). Début 2013, il y avait plus de 700 formations enseignées en anglais, en particulier dans le domaine de la gestion et des sciences de l'ingénieur. Sciences Po et les meilleures écoles de commerce (Essec, HEC) ont ouvert la voie pour ce passage à l'anglais, il y a une dizaine d'années, les autres ont suivi et sont devenues des business schools : ESC Rennes School of Business, France Business School (née de la fusion de l'ESCEM Tours-Poitiers et des ESC Amiens, Clermont et Brest), Euromed Management à Marseille ("Get ready to love your future »), Skema Business School avec des campus à Paris, Lille, Nice, Raleigh et Suzhou, etc. La liste est longue et on craindrait d'ennuyer le lecteur à toutes les citer. Dans l'article du Nouvel Observateur mentionné plus haut, Bruno Sire, président de l'Université Toulouse I - Capitole, reconnaît, beau joueur, que « le ministère a toujours fait preuve de pragmatisme et d'intelligence avec nous " (Gonzague 2013). Il faut savoir que l'Université de Toulouse I abrite en son sein la très sélective Toulouse School of Economics, où une part importante des cours sont

3. Devise de l'école, en anglais dans le texte of course. 
dispensés en langue anglaise. Dans une autre interview, le même Bruno Sire, entre aveu et justification, déclarait : "J'enfreins la loi Toubon tous les jours car elle n'est pas adaptée au monde moderne. » Gérard Duchêne, responsable $\mathrm{du}$ Master of Development Economics and International Project Management à Paris-Est-Créteil, pour sa part, est indigné : "Quand une loi est grotesque comme l'est la loi Toubon, c'est le devoir du citoyen de l'ignorer ! (Brafman 2013).

Dans la série des dérives constatées quant à l'application de la loi Toubon, on ne peut manquer de signaler le cas de Campus France. L'agence gouvernementale pour la promotion de l'enseignement supérieur français proposait, bien avant le début des discussions sur l'article 2 de la loi ESR, une rubrique entièrement consacrée aux formations enseignées en anglais qui explique très clairement aux étudiants étrangers " [qu'] il n’est pas nécessaire de parler français pour étudier en France " (avec quelques fautes d'orthographe que nous n'avons pas cru bon de reproduire ici mais qui réjouiront sans doute les amateurs de mauvais esprit). En Inde, l'un des marchés les plus prometteurs en matière de mobilité étudiante, Campus France, organise des campagnes promotionnelles trimestrielles dans les principales villes du pays (Admission Tour) en avertissant sur son site les établissements français qui souhaitent y participer qu'elle accorde « la priorité aux offres de formation en anglais, les seules véritablement attractives pour le public indien ${ }^{4} .{ }^{4}$ On veut bien le croire et on serait presque tenté de saluer le dynamisme de l'agence et sa bonne connaissance du terrain. Est-il pour autant acceptable que le cadre légal soit purement et simplement ignoré ?

Les chercheurs et les laboratoires de recherche, en France, sont loin de donner un meilleur exemple. En dépit de l'existence du fonds Pascal, fonds créé en 2005 pour soutenir l'interprétariat vers le français dans les colloques scientifiques internationaux, 90 pour cent des rencontres organisées en 2012-2013 dans le domaine des sciences dures l'ont été sans interprète, 27 pour cent en sciences humaines (Héran 2013:3).

Pour terminer ce tour d'horizon, on consultera avec profit le rapport de Dominique Gillot et Ambroise Dupont sur le bilan de "l'autonomie des universités depuis la loi LRU », où les rapporteurs signalent pour mieux les excuser les infractions à la loi Toubon : « Des établissements, dans leur attention particulière pour faciliter l'insertion des étudiants étrangers non francophones, ont développé des cursus en langue étrangère, avec un risque juridique

4. Campus France, disponible sur http://www.campusfrance.org/fr/ [Site régulièrement consulté à partir de février 2012. Dernière consultation, le 21 novembre 2013.] 
avéré pour non-respect de la législation française concernant le monopole du français comme langue de l'enseignement " (Sénat français 2013). Geneviève Fioraso ne dira pas autre chose. Durant une interview dans l'émission Bourdin \& Co sur RMC, la ministre reconnait sans détour que son texte doit permettre aussi de "régulariser ce qui se fait aujourd'hui parce que les écoles [et] les universités qui le font » sont " en infraction avec la loi Toubon ». ${ }^{5}$

Il arrive qu'une loi votée reste non appliquée, faute de moyens ou de volonté réelle de la mettre en application. Il est, en revanche, plus rare de voir une loi, bien connue de l'opinion publique, délibérément ignorée. D’autant plus que dans ce cas précis, les moyens ne font pas défaut. On a mentionné plus haut l'existence du fonds Pascal. Quant au budget de Campus France, il s'élève à $€ 28$ millions, dont plus de $€ 4$ millions de subvention publique (en 2013), pour faire la promotion des formations universitaires en français mais aussi en anglais. En Inde, pour chaque session des Admission tours, les établissements français qui souhaitent y participer déboursent entre $€ 2200$ et $€ 4400$ de frais d'inscription pour faire connaître auprès des étudiants indiens des offres de formations principalement dispensées en anglais. De plus, comme le rappelle François Grin :

L’usage de l'anglais à certaines étapes de la recherche ne justifie en aucune façon qu'une faculté universitaire sise dans un pays francophone prétende n'offrir que des MA principalement, voire entièrement, en anglais. N'oublions pas que le public et les usagers, ce sont aussi les contribuables qui financent l'université, et ils ont le droit de demander à celle-ci d'enseigner dans leur langue. (Grin $2013: 281$ )

Mais la non-application n'était apparemment pas suffisante aux yeux des détracteurs de la loi Toubon qu'ils semblent considérer pour certains comme une insulte personnelle, aussi a-t-il fallu commencer à envisager sérieusement une possible régularisation de la situation, c'est-à-dire supprimer les limitations de vitesse pour éviter aux chauffards d'avoir à payer une amende. Et c'est donc là qu'intervient l'article 2 de la loi ESR.

\section{L'Article 2 de la loi ESR et les modifications du cadre légal}

Pour une vue d'ensemble des évolutions du texte de l'article 2 de la loi ESR visant à modifier le cadre fixé par la loi Toubon, on consultera le tableau suivant: ${ }^{6}$

5. RMC émission du 14 mai 2013.

6. Reproduit avec l'aimable autorisation de M. le Député P. Amirshahi (PS). 
Tableau 1. L'Article 2 et ses modifications

\begin{tabular}{|l|l|}
$\begin{array}{l}\text { L'Article présenté dans le projet de loi de } \\
\text { Geneviève Fioraso : }\end{array}$ & $\begin{array}{l}\text { L'Article après qu'il ait été amendé à l'Assemblée } \\
\text { Nationale: }\end{array}$ \\
\hline
\end{tabular}

Art. 2 Après le premier alinéa du II de l'article L. 121-3, est inséré un alinéa ainsi rédigé :

«Des exceptions peuvent également être justifiées par la nature de certains enseignements lorsque ceux-ci sont dispensés pour la mise en oeuvre d'un accord avec une institution étrangère ou internationale tel que prévu à l'article L. 123-7 ou dans le cadre d'un programme européen. »
Art. 2 Après le premier alinéa du II de l'article L. 121-3, est inséré un alinéa ainsi rédigé :

« Des exceptions peuvent également être admises pour certains enseignements lorsqu'elles sont justifiées par des nécessités pédagogiques (emphasis added) et que ces enseignements sont dispensés dans le cadre d'un accord avec une institution étrangère ou internationale tel que prévu à l'article L. 123-7 ou dans le cadre d'un programme européen et pour faciliter le développement de cursus et de diplômes transfrontaliers multilingues. Dans ces hypothèses, les formations ne peuvent être que partiellement proposées en langue étrangère (emphasis added). Les étudiants étrangers auxquels sont dispensés ces enseignements bénéficient d'un apprentissage de la langue française. Leur niveau de maîtrise de la langue française est pris en compte pour l'obtention du diplôme. »

Art.2 bis Dans un délai de deux ans à compter de la promulgation de la présente loi, le Gouvernement remet au Parlement un rapport évaluant l'impact de l'article 2 sur l'emploi du français dans les établissements publics et privés d'enseignement et sur l'évolution de l'offre d'enseignement du français langue étrangère à destination des étudiants étrangers.

Le texte de l'article 2 du projet de loi a donc été modifié dans un premier temps (Amendement no. 284) pour renforcer le caractère exceptionnel et partiel des formations en langues étrangères. Est-ce pour autant un bon compromis? On peut le penser dans la mesure où les deux arguments mis en avant par les partisans de la libéralisation de la politique linguistique des universités, à savoir la difficulté pour les étudiants non francophones à suivre dès les premiers mois de leur séjour en France des cours en français et l'obligation qui leur serait faite d'apprendre la langue française, sont désormais pris en compte et encadrés par la loi. Nul n'ignore que les promesses des politiciens n'engagent que ceux qui y croient, le fait de les inscrire dans le texte légal constitue donc un progrès et une garantie, ou du moins une base solide pour les futurs débats qui ne manqueront pas de se produire à ce sujet. Au regard des nombreux cas de non application de la loi Toubon, on peut cependant nourrir quelques doutes quant au respect de ces dispositions dans l'avenir. Par ailleurs, le 
gouvernement doit présenter au Parlement une évaluation de l'impact de ces modifications du cadre légal dans les deux ans suivant la promulgation de la loi, ${ }^{7}$ ce qui est en soi une bonne chose mais, du fait de l'existence d'un nombre significatif de formations en anglais dans les universités et les écoles françaises (ainsi que dans d'autres pays européens, en particulier en Allemagne), on peut s'étonner et regretter qu'une première évaluation n'ait pas précédé les débats relatifs à l'adoption du projet de loi ESR. À cet égard, les rapports de la Cour des comptes sur la gestion de Sciences Po (2012) et des grandes écoles de commerce (en 2013) montrent assez clairement que l'internationalisation des établissements supérieurs, qui inclut la mise en place de formations en anglais ou en langue étrangère, a un coût financier très important (recrutement de professeurs ayant une réputation internationale dans leur spécialité, modernisation des locaux et campagne de promotion pour attirer des étudiants étrangers) et place ces établissements dans une situation d'hyper-compétition qui profite seulement aux meilleurs d'entre eux, en termes d'image, de qualité d'enseignement ou de rentabilité pure et simple. Pour les autres, c'est fusion et enseignement low cost.

Après un autre passage devant le Sénat et une réunion de la commission mixte paritaire (composée de sept députés et sept sénateurs permettant d'aboutir à un texte commun des deux assemblées dans le cadre d'une procédure accélérée mise en oeuvre par le gouvernement), le projet de loi ESR, ou loi Fioraso, est devenu la Loi no. 2013-660 du 22 juillet 2013 relative à l'enseignement supérieur et à la recherche (Gouvernement français 2013). L'article 2 modifie le texte de l'article L. 121-3 du Code de l'Éducation de la manière suivante :

I. - La maîtrise de la langue française et la connaissance de deux autres langues font partie des objectifs fondamentaux de l'enseignement.

II. - La langue de l'enseignement, des examens et concours, ainsi que des thèses et mémoires dans les établissements publics et privés d'enseignement est le français. Des exceptions peuvent être justifiées :

$1^{\circ}$ Par les nécessités de l'enseignement des langues et cultures régionales ou étrangères ;

$2^{\circ}$ Lorsque les enseignants sont des professeurs associés ou invités étrangers ;

$3^{\circ}$ Par des nécessités pédagogiques, lorsque les enseignements sont dispensés dans le cadre d'un accord avec une institution étrangère ou internationale tel que prévu à l'article $\mathrm{L}$. 123-7 ou dans le cadre d'un programme européen ;

$4^{\circ}$ Par le développement de cursus et diplômes transfrontaliers multilingues.

7. Trois ans dans la version finale. 
Dans ces hypothèses, les formations d'enseignement supérieur ne peuvent être que partiellement proposées en langue étrangère et à la condition que l'accréditation concernant ces formations fixe la proportion des enseignements à dispenser en français. Le ministre chargé de l'usage de la langue française en France est immédiatement informé des exceptions accordées, de leur délai et de la raison de ces dérogations.

Les étudiants étrangers bénéficiant de formations en langue étrangère suivent un enseignement de langue française lorsqu'ils ne justifient pas d'une connaissance suffisante de cette dernière. Leur niveau de maîtrise suffisante de la langue française est évalué pour l'obtention du diplôme.

Les enseignements proposés permettent aux étudiants francophones d'acquérir la maîtrise de la langue d'enseignement dans laquelle ces cours sont dispensés.

Les écoles étrangères ou spécialement ouvertes pour accueillir des élèves de nationalité étrangère, ainsi que les établissements dispensant un enseignement à caractère international, ne sont pas soumis à l'obligation prévue au premier alinéa.

On le voit, les modifications apportées par l'Assemblée nationale sont non seulement conservées dans la version finale, mais s'en trouvent également renforcées puisque la loi formule distinctement quatre types d'exceptions. Les deux premiers, déjà présents dans le Code de l'Éducation, ne sont pas modifiés. Le quatrième type d'exceptions réaffirme l'attachement de la France à une politique multilingue et aux réalités des échanges avec ses proches voisins européens. En ce qui concerne le troisième type d'exceptions, celui qui touche précisément à la mise en place de formations en anglais dans les universités et dans l'enseignement supérieur français en général, on ne peut que constater que si la notion de nécessité pédagogique semble constituer un sérieux garde-fou face à la tentation du tout-anglais, elle laisse au ministre et aux associations chargées de la défense de la langue une grande marge d'appréciation et une lourde responsabilité. D'où l'importance également du rapport que le gouvernement devra remettre aux commissions permanentes de l'Assemblée nationale et du Sénat qui seul permettra d'évaluer les effets du texte. Son importance est telle d'ailleurs qu'il fait à lui tout seul l'objet de l'article 3.

La loi 2013-66o ayant été promulguée le 22 juillet 2013 par le président de la République, on peut donc espérer la remise d'un rapport sur l'impact des formations en langue étrangère dans l'enseignement supérieur pour juillet 2016. En attendant, il sera toujours possible d'observer l'évolution en temps 
réel de l'offre de formations en anglais sur le site de Campus France. Lagence gouvernementale en dénombrait 810 à la rentrée 2013.

\section{Conclusion : Quand les Brésiliens apprendront l'anglais pour étudier en France}

Dans un article récent, Maria Teresa Zanola déplore l'absence de réflexion sur la politique linguistique dans les universités italiennes ou à l'échelon national. Elle s'interroge sur la demande supposée de cours en anglais des étudiants étrangers $(2013: 251)$ : " qu'est-ce qui nous empêche de penser que des étudiants issus de pays romanophones - venant d'Amérique latine, par exemple, hispanophones ou lusophones, ou d'Europe de l'Est (la Roumanie) - pourraient préférer suivre un second cycle [universitaire] en italien, tout en suivant à côté de cela des cours en anglais? ${ }^{8}$ En effet, il faut rappeler que l'anglicisation des universités, leur américanisation en fait, n'est pas ou ne devrait pas être synonyme d'internationalisation.

La France est depuis longtemps la troisième destination mondiale pour les étudiants étrangers. Qu'elle soit quatrième ou cinquième, ou sixième, ne revêt d'ailleurs qu'une importance secondaire. L'enseignement supérieur en France étant gratuit (ou quasi-gratuit si l'on tient compte des frais d'inscription administratifs annuels : $€ 183$ en licence, $€ 254$ en master et $€ 388$ en doctorat) et cette gratuité s'appliquant à tous les inscrits indépendamment de leur nationalité, la population étudiante étrangère coûte à l'État français la bagatelle de $€_{2}$ milliards à $€_{3}$ millards chaque année (près de $€_{300}$ millions pour les seuls étudiants chinois). Dans ces conditions, on comprend mal pourquoi la France cherche à augmenter sa "part de marché " face à des concurrents qui ne jouent pas au même jeu et pour qui ces étudiants étrangers sont une source de bénéfices, ${ }^{9}$ à moins d'avoir une politique de coopération scientifique ou

8. Citation originale (en anglais) : «Bearing all that in mind, why shouldn't we think that students of romance languages - coming from South America, for example, from the Portuguese or Spanishspeaking world, or from Eastern Europe (e.g. Romania) - would prefer to have a second cycle in Italian, while learning English at the same time? »

9. Environ 15 milliards d'euros pour l'Australie, en 2012-2013, soit le quatrième poste de la balance commerciale du pays. Dans son étude sur la Réforme de l'enseignement supérieur (en France), P.-A. Chiappori (2011 : 50), professeur d'économie à Columbia University (New York), n'en revient toujours pas : «Que les établissements français consacrent une part non négligeable de ressources largement insuffisantes à former gratuitement des dizaines de milliers d'étudiants chinois dépasse l'entendement - d'autant que rien ne garantit que ces étudiants soient d'un niveau particulièrement élevé. » 
des objectifs diplomatiques bien définis, ce qui n'est malheureusement pas le cas. Que certaines écoles de commerce ou Sciences Po aient adopté une telle politique d'internationalisation, cela peut se concevoir puisque ce positionnement stratégique sur un créneau d'habitude réservé aux établissements américains s'est accompagné d'une hausse conséquente des frais de scolarité. Pour les universités, il faudrait ouvrir un débat sur la fin de la gratuité et sur la privatisation de l'Enseignement supérieur, sujet autrement plus délicat que celui de la langue d'enseignement et que les gouvernements successifs, depuis une trentaine d'années, ont réussi à escamoter en pratiquant une politique des petits pas. Par ailleurs, les étudiants marocains ou sénégalais, et les autres francophones qui choisissent précisément la France pour pouvoir étudier en français sont-ils moins « internationaux » que les étudiants chinois ou indiens ?

On a entendu certains défenseurs de l'anglicisation du supérieur se risquer à de sombres prédictions : si nous ne faisons pas de cours en anglais, les étudiants des pays émergents ne viendront pas chez nous. Et de citer dans le désordre : l'Inde, la Chine, la Corée du Sud (?) et le Brésil. On ne s'étendra pas sur la présence de la Corée du Sud dans la liste des pays émergents (si ce n'est pour souligner que les étudiants coréens sont, il est vrai, parmi les plus mobiles du monde selon les indicateurs de l'OCDE (2012)). En revanche, la mention du Brésil a de quoi laisser songeur. Si demain des étudiants brésiliens viennent suivre des cours dans des universités françaises parce qu'ils sont en anglais, connaissant la proximité du portugais et du français, il faudra sérieusement s'interroger sur ce désir un peu trouble de s'angliciser à tout prix. Comme le fait remarquer François Grin (2013 : 280), "l'obsession de 'l'étudiant étranger' amène à des absurdités ". Le champ de recrutement, le vivier de candidats potentiels, qui s'ouvre à l'enseignement supérieur français est pourtant immense : étudiants francophones d'Afrique, d'Europe, d'Amérique, hispanophones, lusophones, italophones, apprenants de français du monde entier.

L'existence d'un cadre linguistique légal, pour imparfait qu'il soit dans sa conception comme dans son application, a au moins l'immense mérite de ne pas laisser aux seuls " experts », c'est-à-dire principalement les dirigeants d'établissements supérieurs, privés ou publics, ou à des ministres qui apparaissent de plus en plus comme de simple porte-paroles de ces mêmes experts, le soin de décider de ce qu'est une langue (de communication, de culture, d'enseignement), de ce que chacun est prêt à sacrifier sur l'autel de la mondialisation économique et de ce qui doit faire ou pas l'objet d'un débat démocratique. 


\section{Références}

Beaudin, H. (2010). 'La Dérive linguistique dans l'enseignement supérieur français'. Disponible en ligne sur : http://www.forumpourlafrance.net [consulté le 11 novembre 2013].

Chiappori, P.-A. (2011). 'Réforme de l'enseignement supérieur : quelle place pour les entreprises?' Nancy: Institut de l'entreprise.

Eloy, J. M. (1997). « Aménagement » ou «politique » linguistique? Mots, 52.1 : 7-22.

Grin, F. (2013). 'L'Anglais dans l'enseignement académique : le débat s'égare dans les clichés'. European Journal of Language Policy/Revue europénne de politique linguistique, $5.2: 279-83$.

Hagège, C. (2012). Contre la pensée unique. Paris : Odile Jacob.

Héran, F. (2013). L'Anglais hors la loi? Enquête sur les langues de recherche et d'enseignement en France (no. 501). Paris : Institut National d'Études Démographiques (INED).

Truchot, C. (2008). Europe : l'enjeu linguistique. Paris : La Documentation Française.

Zanola, M. T. (2013). 'The Attractiveness of Second-cycle and PhD Studies in Europe - A Question Among Others: Which Language?' European Journal of Language Policy/ Revue europeénne de politique linguistique, 5.2, 247-56.

\section{Textes officiels}

Académie française (2013). Déclaration du 21 mars 2013. Disponible en ligne sur :http:// www.academie-francaise.fr/actualites/declaration-de-lacademie-francaise-du21-mars-2013 [consulté le 11 novembre 2013].

Ayrault, J.-M. (2013). Circulaire du 25 avril 2013 relative à l'emploi de la langue française, adressée aux ministres par J.-M. Ayrault, premier ministre. No. 5652/SG. Disponible en ligne sur : http://www.dglf.culture.gouv.fr/droit/Circulaire\%2on\%C2\%Bo\%2O 5652-SG\%20du\%2025\%20avril\%202013.pdf [consulté le 11 novembre 2013].

Gouvernement français (2013). Loi no. 2013-66o du 22 juillet 2013 dite loi Fioraso relative à l'enseignement supérieur et à la recherche (ESR), articles 2 et 3. Disponible en ligne sur : http://www.legifrance.gouv.fr

Gouvernement français (2014a). Code de l'Éducation, articles L. 121-23 ; L. 121-3. Disponible en ligne sur : http://www.legifrance.gouv.fr

Gouvernement français (2014b). Loi no $94-665$ du 4 août 1994 dite Toubon relative à l'emploi de la langue française. Disponible en ligne sur : http://www.legifrance.gouv.fr

\section{Rapports}

Attali, J. (1998). Pour un modèle européen d'enseignement supérieur (1998), rapport rédigé sous la direction de J. Attali.

Cour des comptes (2012). Sciences Po : une forte ambition, une gestion défaillante. Rapport de la Cour des comptes. 
Cour des comptes (2013). Les écoles supérieures de commerce et de gestion : un développement à réguler. Rapport de la Cour des comptes.

OCDE (2012). Regards sur l'éducation 2012 : les indicateur de l'OCDE. Étude de l'OCDE. Paris : L'Organisation de Coopération et de Développement Économiques.

OCDE (2013). Regards sur l'éducation 2013 : les indicateur de l'OCDE. Etude de l'OCDE. Paris : L'Organisation de Coopération et de Développement Économiques.

Sénat français (2013). L'autonomie des universités depuis la loi LRU : le big-bang à l'heure $d u$ bilan. Rapport d'information rédigé par D. Gillot et A. Dupont, sénateurs. Paris : Parlement français.

\title{
Articles de presse
}

Arnaud, P. (2013). La Mort du français à l'université. Transcription de l'interview de Michel Serres et Claude Hagège par David Pujadas pour le journal télévisé de France 2 du 15 mais 2013. Le Grand Soir, 18 mai.

Attali, J. (2013). 'Enseigner en français !' L’Express, blog hébergé, 22 avril. Disponible en ligne sur : http://blogs.lexpress.fr/attali/2013/04/22/enseigner-en-francais/ [consulté le 11 novembre 2013].

Brafman, N. (2013). J'enfreins la loi Toubon tous les jours car elle n'est pas adaptée au monde moderne. Interview de B. Sire, président de l'Université Toulouse I Capitole. Le Monde, blog hébergé, 22 mai. (consulté le 11 novembre 2013).

Cerquiglini, B. (2013). Ne nous laissons pas abuser par des illusions marchandes. L'Humanité. $f r, 26$ avril. (Consulté le 11 novembre 2013).

Compagnon, A. (2013). Un amour de Mme Fioraso. Libération, 3 avril. (Consulté le 11 novembre 2013).

Garçon, F. (2013). Fioraso veut des cours en anglais à la fac : évidemment, c'est une bonne idée. Le Nouvel Observateur, 16 mai [consulté le 11 novembre 2013].

Gonzague, A. (2013). Cours 100\% en anglais à la fac : ça se fait déjà, my dear ! Le Nouvel Observateur, 19 avril. (Consulté le 11 novembre 2013).

\begin{abstract}
The Law of 4 August 1994 on the use of the French language, better known as the Toubon law, was passed to protect consumers, workers and cultural creation in French in the face of the growing dominance of English and of American culture in Europe. The scope of the Toubon law also extends to education, higher education and research. In April and May 2013, Geneviève Fioraso, Minister of Higher Education and Research, presented to the French Senate and the National Assembly a plan to reform universities, the second article of which sparked a heated controversy. By expanding the range of authorised exceptions to the provisions of the Toubon law that would allow French academic institutions to implement courses taught in a foreign language, that is, in English, Article 2 threatened to undermine the fundamental principle of the Toubon law. The changes in the legal-linguistic context in France that would be required to overcome difficulties in enforcing the law, together with
\end{abstract}




\section{Jean-François Graziani}

the need to address the problem of existing courses taught in English at French universities, illustrate - perhaps more clearly than other factors - the new issues facing universities in the current situation of hyper-competition on a global scale.

Keywords: English-medium instruction; anglicisation; French language; higher education; Toubon law; Fioraso law 